\title{
Thomas Ots
}

Graz, Österreich

\section{Neuer Wein in neuen Schläuchen!}

Liebe Leserinnen und Leser,

inzwischen haben Sie die neue, bei Springer Medizin verlegte DZA bereits einmal in den Händen halten können. Was wir von vielen von Ihnen gehört haben, ist allgemein großes Lob: Die DZA ist besser lesbar und interessanter geworden. Auch Akupunkteure altern in einem Jahr um 365 Tage, und die größere Schrift sowie das aufgelockerte Layout verführen dazu, die DZA gleich mal schnell - und auch zwischendurch - zu lesen. Haben Sie sie in den letzten Jahren mit zunehmend kleiner werdender Schrift nicht manchmal zur Seite gelegt, um sie hinterher „in Ruhe "durchzuschauen, und wie oft fiel Ihnen dieses Ritual dann wieder ein, als Sie eben diesen Stapel mit demselben Gedanken um eine neue Ausgabe bereicherten?

\section{Zum Inhaltlichen: Wissenschaft}

„DZA Neu“ bedeutet, dass vieles, das sich bewährt hat, fortgeführt wird, aber einige Schwerpunkte haben sich verändert. Wissenschaft: Wir werden uns stärker als zuvor bemühen, Originalia zu bringen. Garant hierfür sind die universitären Anbindungen des Rubrikenverantwortlichen Johannes Fleckenstein sowie der zwei Neuen in der Redaktion: Heidemarie Haller und Janina Geib (insgesamt wurde durch unseren Aufruf letztes Jahr die Redaktion deutlich vergrößert und auf breitere Beine gestellt - es sind zu viele, um sie hier alle aufzuführen, Sie finden sie aber auf der Seite „Redaktion“). Die einzelnen die DZA tragenden Gesellschaften sind jetzt gleichmäßiger vertreten, und außerdem haben wir auch Redaktionsmitglieder gewinnen können, die nicht einer Akupunkturgesellschaft angehören.

Zur Rubrik Wissenschaft gehören auch der Journal Club und die Serie „Wissenschaft für die Kitteltasche (in Österreich: Manteltasche)“. Vielleicht sollte dieser Titel noch einmal erklärt werden. Wie wir immer wieder sehen, werden alternative, komplementäre und integrative medizinische Sichtweisen von einigen Menschen, die sich dazu berufen fühlen, angegriffen. Ob bewusst oder unbewusst, wird doch immer wieder behauptet, die Akupunktur sei nicht besser als Placebo, es gäbe auch keine Studien etc. (https://www.focus.de/gesundheit/chronische-schmerzenforscher-streiten-ueber-nutzen-und-wirkung-von-akupunktur_id_8632751.html). Diese Gegner beharren auf einem falschen Placebobegriff, denn bei jeder Form eingeschränkter Akupunktur bzw. Akupressur, mit richtigen Nadeln oder dem "Theaterdolch", handelt es sich nicht um Placebo, sondern um Sham $=$ nicht regelkonform ausgeführte, aber wirksame Therapie. Und so wird geflissentlich übersehen, dass sich Verum- und Sham-Akupunktur in dreiarmigen Studien nur wenig unter-

Deutsche Zeitschrift für Akupunktur

2018 • 61 (2): 59-60

https://doi.org/10.1007/s42212-018-0053-8

Online publiziert: 16. April 2018

๑) Springer Medizin Verlag GmbH, ein Teil von Springer Nature 2018 schieden, aber beide in manchen Belangen der konventionellen Therapie deutlich überlegen waren. Und doch sind es immer wieder angebliche „Kenner der komplementären Therapien“ wie Edzard Ernst, die in der Öffentlichkeit mit ihren Urteilen aufscheinen und in diese Trompeten mit ihren falschen Tönen blasen. Zum Trost: Die Leugnung des Klimawandels durch einen anderen "großen Mann“ hat für die Menschheit wesentlich fatalere Konsequenzen als die Leugnung der Akupunkturerfolge seitens Edzard Ernst.

Die Frage der Wahrheit in der Medizin ist nur zum Teil eine wissenschaftliche, ganz wesentlich aber eine der Macht. Wer bestimmt über die Meinung der Öffentlichkeit? Welche negativen Medien werden in den asozialen Medien oder Leserkommentaren gezielt verbreitet? Erst kürzlich sah ich Kommentarschreiber mit über 8000 Einträgen. Honi soit qui mal y pense. Gegen die allgemeine Meinung anzureden ist unheimlich schwer, aber wir haben auch eine Macht. Wir können uns die kritisch observierten Nachweise der Akupunktur aus der DZA kopieren und in die Kitteltasche stecken. Ein Tipp: Wenn Ihnen demnächst wieder ein kritischer Kollege erzählt, dass Akupunktur Placebo sei, dann fragen Sie ihn bitte: „Wie viele randomisierte kontrollierte Akupunkturstudien, also gemäß EBM, gibt es? 50, 100 oder 200?" Die meisten entscheiden sich für eine dieser drei Zahlen. Dann sagen Sie ihm: „Allein seit 2017 sind es 3960“ (PubMed-Abfrage 25.03.2018). Und dann drücken Sie dem Kollegen, der daraufhin seinen Mund kaum zubekommt, eine der Serien für die Kitteltasche in die Hand: Überzeugungsarbeit von unten.

\section{Zum Inhaltlichen: Praxis - Schwerpunkt Praxis}

Die meisten von uns sind ja nicht nur Akupunkteure, sondern Allrounder, die unterschiedliche Therapien zum Wohl unserer Patienten anwenden. Und deswegen kamen wir auf die Idee, in unseren Praxisschwerpunkten eine Frage zu stellen und zu schauen, wie diese Frage von unterschiedlichen Seiten aus beantwortet wird. In der letzten Ausgabe war diese die zeitgerechte Frage der Pollinosis mit acht Beiträgen verschiedener Autoren. Das werden wir nicht immer so durchführen (z. B. in dieser Ausgabe, da das Thema des Biss- und Kiefermanagements enormen Platz benötigt), aber immer öfter. Die Rubrikenverantwortlichen für diese Praxisschwerpunkte werden von Frage zu Frage wechseln - auch ein Garant für Lebendigkeit durch Variantenreichtum. Die Praxis-Schwerpunkte der nächsten beiden Ausgaben werden sich den Rückenbeschwerden - immer noch einer der wichtigsten Ursachen von Arbeitsausfällen und Frühberentungen - und dann gastrointestinalen Beschwerden widmen.

\section{Zum Inhaltlichen: Panorama}

In der TCM gibt es drei Gruppen von pathologischen Faktoren: äußere, innere und weder noch. Auch die „DZA Neu“ hat diese Dreiteilung, denn viele Beiträge können nicht unter Wissenschaft oder Praxis eingeordnet werden. Und das sind eben oft Beiträge, die von Ihnen kommen. Sie möchten uns etwas mitteilen, möchten uns auf eine Beobachtung hinweisen, haben etwas 
Neues entwickelt, möchten uns Ihre Meinung zu einem Trend mitteilen, haben eine Frage etc. Ja, bitte! Wir möchten Sie ausdrücklich auf diese Möglichkeiten hinweisen. Schreiben Sie uns. Leserbriefe und eingereichte Artikel, kurz oder in Artikellänge, sollten von der Lebendigkeit zeugen, die die DZA unter ihren Lesern anstößt. Seien Sie kritisch und halten Sie sich nicht mit Kritik zurück!

Und wie oft machen wir überraschende und begeisternde Erfahrungen mit unseren Patienten, vor allem auch mit denen, die - sozusagen „austherapiert“ - uns als „letzte Hoffnung“ aufgesucht haben. Fallberichte sind eine großartige Möglichkeiten, die Diskussion zu bereichern, abgesehen davon, dass Expertenurteil in der EBM ebenfalls seinen wichtigen Platz hat.

\section{Zukünftige Fragestellungen}

Nicht nur in der Ohr-Akupunktur gibt es - wie der leider vor einem Jahr verstorbene Marco Romoli es formulierte (DZA 1/2017), großen Forschungsbedarf, auch in der Körper-Akupunktur bedarf es noch großer Anstrengungen, ihre Theoriemodelle aus dem Bereich verbliebener Metaphern in die Welt naturwissenschaftlicher Erklärungen zu transferieren. Eine Aufgabe, die sich die DZA mit großer Intensität widmen bzw. fortsetzen wird, ist die Aufarbeitung der Frage, inwieweit die Wirkwege der Akupunktur auf die Segment-Struktur des Körpers zurückgeführt werden können: Segment-Anatomie. Hierzu finden Sie in dieser Ausgabe einen Artikel, der sich die Frage stellt, was ei- gentlich passiert, wenn wir den Akupunkturpunkt Dickdarm 4 reizen. Weiterhin finden Sie auch in dieser Ausgabe einen Artikel zur Bedeutung der Faszien für die Akupunktur. Und einen weiteren darüber, wie Nadelstiche von uns subjektiv aufgenommen werden (Therapeutische Empfindungen). Denn wir sind uns sicher, dass es nicht nur einen einzigen Wirkweg der Akupunktur gibt, sondern mehrere, die alle eine Gemeinsamkeit haben: den Druck oder Stich auf/in ein bestimmtes Körperareal, eine bestimmte Körperstruktur.

Es bleibt also spannend. Deswegen möchte ich auch alle alten und neuen Mitglieder unserer großen Redaktion für Ihre Bereitschaft danken, sich in diese zukünftigen Diskussionen einzubringen.

Ich verbleibe mit dem schon öfter von Eiffe geborgten Slogan: „Bis hierher und noch weiter!“

Ihr Thomas Ots

\section{Korrespondenzadresse}

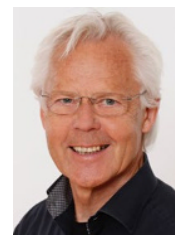

DDr. Thomas Ots

Burgring 8, 8010 Graz, Österreich

ots@daegfa.de

Interessenkonflikt. T. Ots gibt an, dass kein Interessenkonflikt besteht.

\section{Ihr Online Zugang}

Lesen Sie die Deutsche Zeitschrift für Akupunktur online auf www.springermedizin.de

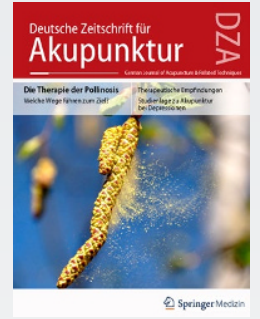

Als Abonnent der DZA kön-

nen Sie die Zeitschrift auf

www.springermedizin.de/dza ab sofort auch online lesen. Mit dem neuen e-Paper können Sie die DZA außerdem jederzeit bequem auf Ihrem Tablet lesen.
Sind Sie bereits bei SpringerMedizin.de registriert? Dann wird Ihr Zeitschriftenabonnement automatisch Ihrem Online-Nutzerkonto hinzugefügt. Sollten die Angaben Ihres Online-Accounts nicht eindeutig mit den Angaben Ihres Zeitschriften-Abonnements übereinstimmen, kann die Zuordnung nicht sicher erfolgen. In diesem Fall und bei allen anderen Fragen zum Online-Zugang kontaktieren Sie bitte unseren Kundenservice unter: kundenservice@springermedizin.de

So einfach erhalten Sie Zugang zum Online-Archiv:

- Registrieren Sie sich einmalig auf

www.springermedizin.de/register

- Geben Sie Ihre Einheitliche Fortbildungsnummer (EFN) an.

- Ihr Benutzername entspricht Ihrer E-Mail-Adresse, Ihr Passwort können Sie frei wählen und später jederzeit unter "Mein Profil"

Telefonisch erreichen Sie die Hotline montags bis freitags von 9.00 bis 17.00 Uhr kostenfrei unter 0800-77 80777 sowie gebührenpflichtig aus dem Ausland unter +4930884293600 